\title{
Brocado aplicado: fuentes escritas, materiales y técnicas de ejecución
}

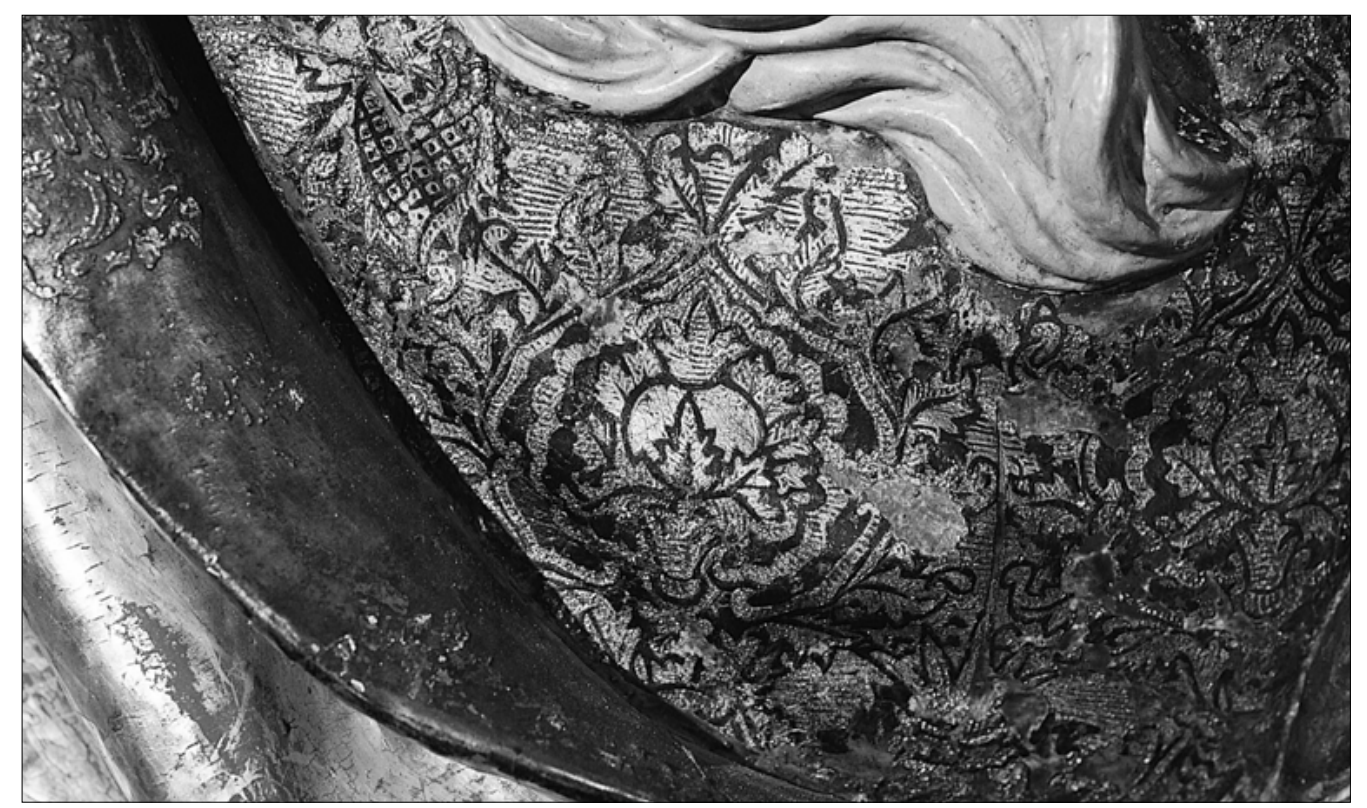

Ma José González López

Conservadora-Restauradora de Bienes

Culturales.

Facultad de Bellas Artes, Universidad de Sevilla

\section{Resumen}

El brocado aplicado es una técnica de decoración pictórica y polícroma originaria de los Países Bajos con un cronología temporal bien delimitada, mediados del siglo XV a mediados del siglo XVI. Se desarrolla por varias de sus escuelas situándose sus inicios alrededor de 1420; de entre ellas destacan las escuelas de primitivos flamencos, la de Colonia, y también, la de Bruselas, en esta última se sigue utilizando hasta 1560. Desde estos lugares, se propaga hacia la zona del Rin, especialmente hacia el Sur de Alemania ge- neralizándose su empleo como decoración polícroma en todo Europa (Austria, Suiza, Lombardía -Alta Italia-, Francia, Portugal y España).

Debe su denominación "brocado aplicado", a la decoración textil cuyas características imita, tejidos de brocados de seda con o sin aplicaciones de bordados con hilos de oro o de plata.

Este artículo intenta delimitar los materiales que intervienen en su realización, el proceso de ejecución, el método de aplicación en la obra y el estado actual de la cuestión en base a los estudios que sobre esta técnica se han realizado en las últimas décadas. Para ello se parte del análisis de las fuentes escritas específicas de esta técnica, en concreto de los tratados y tratadistas que la describen, así como de la bibliografía especializada. Con ello pretendemos contribuir a su divulgación, al igual que aportar nuevos datos que ayuden al mejor conocimiento de esta técnica consi- 
derada como la más desconocida y a la vez más ricas, detallista y preciosista, de entre las conocidas hasta el momento.

\section{Palabras claves}

Técnica polícroma / Brocado aplicado / Brocados yuxtapuestos / Brocados continuos / Piezas de brocados / Motivos aplicados / Matriz / Molde / Masa de impresión / Dorado / Mixtión / Lámina de estaño

\section{Introducción}

El brocado aplicado es una técnica de decoración polícroma originaria de los Países Bajos con una cronología bien precisa, desde mediado del siglo XV a mediado del siglo XVI. Desde estos países se difunde por toda Europa, en primer lugar, en obras de importación o realizadas por artistas de origen centroeuropeo, y posteriormente, en talleres locales que han asimilado la técnica.

Está considerada como la técnica más rica y preciosista de entre las técnicas decoración de la escultura en madera policromada, y también la más desconocida. Las causas de este desconocimiento la tenemos que buscar en factores tan variados como: las incógnitas todavía existentes en relación con su ejecución técnica, los pocos casos de esculturas en los que está documentada con una metodología científica, el mal estado de conservación en el que por lo general ha llegado hasta nuestros días y por último, los inadecuados tratamientos de conservación-restauración sufridos por numerosos ejemplares.

Esta técnica gran desconocida, se difunde en los círculos especializados, historiadores, restauradores y científicos, a partir de 1963 cuando Mojmir Frinta publica varias casos de esculturas en las que aparecen aplicaciones de brocado aplicado que son estudiados con metodología científica. Esta publicación sigue constituyendo hoy día el inicio de su difusión.'

Este articulo intenta contribuir a la divulgación de esta técnica a partir del análisis de las fuentes escritas -tratados y bibliografía especializada-, sin pretender solucionar ni despejar todas las incógnitas que siguen existiendo entorno a ella, sino más bien, haciendo hincapié en la necesidad de continuar su investigación empleando una metodología científica y técnica a fin de poder interrelacionar datos y extraer conclusiones entre todos los especialistas que trabajamos en este campo.

\section{Terminología}

La mayoría de la bibliografía especializada que sobre esta técnica existe se encuentra en alemán, francés e inglés, es por ello que consideramos fundamental exponer los principales acepciones terminológicas en la que podemos encontrarla.
Alemán: Prebrokate, pressbrokate, brokatapplicationen, pressbrokat, pressbrokat applicationen, pressbrokat-application.

Inglés: Appliqué relief brokad, Brocade apllication, pressed brocade, applied brocade.

Francés: Brocart appliqué.

Español: Brocado aplicado.

Su denominación, "brocado aplicado", deriva de la decoración textil cuyas características imita, tejidos de brocados de seda con o sin aplicaciones de bordados con hilos de oro o de plata, es decir "se refiere a las imitaciones de tejidos bordados con hilos de oro y a la técnica de reproducir el relieve dibujado mecánicamente y a encolar el motivo reproducido sobre un objeto" 2.

El objetivo de esta técnica no es otro que imitar la apariencia de los suntuosos tejidos y ropajes realizados en terciopelo de seda que dominaron el mundo de la moda del siglo XV; tejidos en principio reservados para la más elevada jerarquía eclesiástica y del Estado, si bien se popularizan para cierta clase social en este siglo. Estas telas eran empleadas tanto en las vestiduras de estos personajes (vestidos, túnicas, mantos, etc.) como en sus accesorios (cintas, cinturones, bolsas, etc.) y también, en la decoración de sus hogares (tapices, cortinas y cubrecamas).

Estos tejidos de gran calidad empezaron a producirse en los talleres textiles italianos de la segunda mitad del siglo XIV, destacando los talleres de Lucca, Génova Venecia, Milán y Ferrara, siendo imitados posteriormente por los talleres florentinos quienes perfeccionaron la técnica convirtiéndolos en el producto de mayor éxito comercial. Desde estas ciudades se importan a toda Europa 3.

\section{Cronología}

La técnica del brocado aplicado en policromía se desarrolla a principios del siglo XIV ( \pm | 420) por varias escuelas destacando la de primitivos flamencos, la de Colonia, y también, la de Bruselas, donde se sigue utilizando hasta 1560. Desde estos lugares se propaga hacia la zona del Rin, especialmente hacia el Sur de Alemania. Su empleo como decoración polícroma se generaliza en todo Europa desde la mitad del siglo XV hasta la mitad del siglo XVI (Austria, Suiza, Lombardía -Alta Italia-, Francia, Portugal y España). A partir de esta época, la bibliografía consultada parece estar de acuerdo en que cae en desuso.

El periodo tan concreto en el que desarrolla esta técnica permite datar las obras decoradas con ella, tanto en su conjunto (obras que conserven sus características originales y no han sido modificadas en intervenciones posteriores), como su policromía (obras que conserven estas decoración subyacentes a repolicromías posteriores). Hasta el momento se continua afirmando que el primer caso conocido de una obra donde aparece es en la tabla del maestro de Flémalle de 1453 (fragmento de la Pasión), hoy en el Städel de Francfort 4 . 


\section{Tipología de obras}

Los brocados aplicados, también denominados brocados con molde 5 aparecen en las principales manifestaciones artísticas de la época, es decir en obras pictóricas ${ }^{6}$ (fundamentalmente en los fondos de las pinturas sobre tabla y en menor medida en artesonados y pintura mural), y en obras escultóricas (retablo -fondo de los encasamentos y en las esculturas exentas o adosadas a ellos- y también en obras escultóricas: en ambos casos, en lugares precisos de la obra -bordes, cenefas, túnicas, mantos y vestidos de los personajes, etc.-)

\section{Fuentes escritas}

La utilización de la lámina de estaño en la decoración de obras pictóricas y escultóricas se encuentra descrita por tratados y tratadistas desde el siglo IX en adelante (Lucca MS). Como veremos su empleo, con fines decorativos, es muy variado y por lo general cuando se usa dorado, aparece unido al menor coste económico que supone su empleo en lugar del oro. Así vemos descrita cuatro formas diferentes de utilización:

- En blanco (puliendo su superficie).

- Dorado (corlando la cara visible).

- Aplicaciones de motivos decorativos planos (generalmente dorados).

- Aplicaciones de motivos decorativos con relieves (dorados), como ocurre en el caso específico del brocado aplicado.

Si hacemos un breve recorrido por estos tratados vemos como el siglo IX describe su uso el Manuscrito de Lucca y posteriormente en el siglo XII el Mappae Clavicula ${ }^{7}$, en ambos casos se especifica su empleo con aplicación de películas transparentes de color y con esmalte dorado. También en el siglo XII el manuscrito del monje Theophilus 8 indica la utilización de la lámina de estaño corlada como forma de obtener un efecto de dorado a bajo coste, la tipología de obras sobre la que se aplica no se describe, si bien indica, en los casos que se decora con colores, el aceite de linaza como aglutinante de los pigmentos. El efecto dorado se consigue mediante corladura realizada con tallos de aliso o espino, azafrán y vino o cerveza que es aplicada cuando la lámina de estaño ha sido previamente protegida con un barniz a base de aceite de linaza y resina.

Heraclius ${ }^{9}$, un siglo después, describe dos métodos diferentes de utilizar la lámina de estaño; el primero de ellos, estaño dorado mediante corladura (similar al indicado por Teófilo), si bien en este caso la corla está realizada con cerveza y negro de hollín ; y el segundo método, indicado para el estaño en blanco ${ }^{10}$, mediante pulido y posterior bruñido con pedernal de su superficie. Sólo en el segundo método indica su aplicación sobre madera, cuero y cualquier sitio que se quiera dorar.

A finales del S. XIII o principios del S. XIV Pier de Saint Audemar "I indica varios métodos de emplear la lámina de estaño sobre soportes de madera o papel.
Lo interesante de este tratado, desde mi punto de vista, es la descripción que realiza de la puesta en obra de la lámina de estaño dorada a la que previamente se le había dotado de una forma intencionada. Resulta obvio que no se está refiriendo a la técnica del brocado aplicado, pero si a sus posibles variantes, las aplicaciones o motivos aplicados, ya que especifica de forma muy pormenorizada, la manera de realizar motivos decorativos, preconcebidos de antemano, con lámina de estaño dorado, al igual que la forma de aplicarlos sobre obras de madera o papel, siendo muy claro y preciso en la explicación del método:

La hoja de estaño, previamente preparada con agua de goma y con varias capas de una mezcla realizada con flores de azafrán y clara de huevo (untada con los dedos), se pule con piedra ónix o se engrasa con aceite de lino. El dorado de la lámina de estaño se consigue por cuatro métodos diferentes:

Primer método: Aplicando sobre la superficie del estaño el líquido tamizado resultante de la cocción de mirra y áloe (a partes iguales) e introduciendo la lámina de estaño, así preparada en una vasija en la que se haya cocido el interior de la corteza del ciruelo negro.

Segundo método: Sumergiendo la lámina de estaño en una vasija donde se ha cocido previamente aceite de lino y resina (a partes iguales) y barniz. Tras lo cual se saca y se deja secar al sol.

Tercer método: Se aplica sobre el estaño una pasta obtenida mediante la mezcla de dos cocciones diferentes: por un lado con aceite de lino y la parte interna de la corteza del ciruelo negro y por otro, con ámbar (glassa), alumbre, sangre de dragón y resina.

Cuarto método: Mediante la aplicación de una pasta obtenida por la cocción de aceite de lino o de cáñamo y el interior de la corteza de ciruelo negro.

De la forma de aplicar la lámina de estaño cortada en la forma deseada sobre la obra, nos indica que ha de ser dispuesta sobre una masa (extendida en aquellos lugares en los que se va a poner el estaño) realizada con agua de goma y pigmento blanco. Masa que se ha de pulir una vez seca con piedra de ónix, tras lo cual, se aplica agua de goma y se deposita encima la lámina de estaño. Por último se limpia la superficie y se pule con piedra de ónix.

También Cennini describe la forma de realizar aplicaciones o motivos aplicados en pintura mural 12 con lámina de estaño en blanco o dorado con oro fino. En cuanto a la forma de estos motivos deja libertad de elección al artista , no obstante describe tanto la aplicación de estrellas sobre un cielo azul de azurita utilizando como adhesivo una bolita de cera, o aureolas de Santos, en este caso, empleando como adhesivo barniz líquido (método señalado para el estaño dorado con oro fino), como también precisa las aplicaciones de rosetas y otras cositas lindas. Es curioso observar como en todos los casos el motivo se dibuja y se corta con una regla sobre la lámina de estaño extendida sobre 
$\longleftarrow$
$\square$

...una tabla bien alisada de nogal, peral o ciruelo no muy delgada, escuadrada y del tamaño de un folio real..

Del brocado aplicado hasta el momento son dos las fuentes conocidas que hablan de esta técnica de decoración: El libro del arte o Tratado de la Pintura de Ceninno Ceninni. ${ }^{3}$ y El Liber Illuministarius o Manuscrito de Tegersnsee ${ }^{14}$.

Ambas, describen la técnica de aplicaciones realizadas con lámina de estaño obtenidas a partir de una matriz en la que era grabado el motivo a reproducir. Cronológicamente el tratado de Cennini lo podemos situar hacía finales del S. XIV, mientras que el Liber Illuministarius, o manuscrito de Tegersnsee, por las fechas especificadas en su interior, lo podemos ubicar en torno al primer cuarto del S. XVI.

A partir de estas consideraciones nos planteamos la primera incógnita a considerar, ¿cuál es la fuente origi-

Ficha I. TRATADO DE CENINO CENINI

\section{FICHA TÉCNICA:}

BROCADO APLICADO

\section{FUENTE:}

\section{EL LIBRO DEL ARTE 0 TRATADO DE LA PINTURA DE CENNINO CENNINI}

\section{TIPOLOGÍA:}

Técnica adecuada para su empleo en obras pictóricas (pintura mural y pintura sobre tabla) y en la ornamentación de cofres y piedra.

\section{MATERIALES:}

Según su empleo describe los siguientes materiales:

Dorado y masa de relleno:

- Estaño batido en hojas

- Oro fino

- Yeso molido o yeso grueso con cola

- Mixtión

Aplicación:

- Pez de calafate o pez griega.

\section{MÉTODO DE EJECUCIÓN}

Molde: Piedra tallada con el dibujo deseado.

Proceso:

A Se unta la superficie del molde con grasa 0 sebo

B Se coloca encima la hoja o pan de estaño batido

C Sobre el estaño se dispone estopa mojada.

D Se golpea cuanto puedas con una maza de sauce por el lado de la estopa.

E Se elimina la estopa

F Se rellena con una espátula de madera el hueco de la impronta dejada en la hoja de estaño con yeso grueso molido y cola.

Dorado:

Se extiende el mordiente encima del estaño y cuando está a punto se dora con oro fino.

Aplicación: Sólo indica el método de aplicación sobre muro, pegándolo a éste con pez de calafate "pece de nave", pez griega. naria de la técnica del brocado aplicado? ya que la bibliografía especializada indica por lo general el manuscrito de Tegersnsee como la fuente tradicional de este técnica y no el tratado de Cennini.

\section{El libro del arte o tratado de la pintura de Cennino Cennini}

Shlosser en su Literatura artística sitúa este tratado alrededor de 139015 si bien la época precisa en que se escribe es desconocida. De este tratado existen tres códices en las Bibliotecas Laurentina, Riccardina y Vaticana respectivamente, aunque no se sabe con precisión cual de ellos es el original, si sabemos que de los tres, el único que está datado es el Laurentino, fechado el 31 de junio de 1437.

Cennini, alumno de los maestros Agnolo y Taddeo Gaddi y por tanto del Giotto, formula en su tratado de manera muy descriptiva y estructurada la tradición corporativa de los talleres de arte del S. XIV en Florencia y Padua.

La técnica del brocado la encontramos descrita en su capítulo CXXVIII: De cómo se hacen algunos relieves y cómo sirven para el muro y la tabla.

Las dos versiones consultadas de este tratado plantean algunas diferencias ${ }^{16}$ que no alteran sustancialmente la descripción del método. Estas discrepancias se concretan en la denominación del capítulo y en la naturaleza del yeso utilizado en la masa de relleno. ${ }^{17}$

Los datos de interés sobre esta técnica descritos en este tratado se encuentran resumidos en la fichal.

\section{El Liber Illuministarius o manuscrito de Tegernsee}

El Liber Illuministarius, o códice germánico 821 es uno de los manuscritos del S. XV encontrados en la Biblioteca de Munich procedente del monasterio de Tegernsee. Este manuscrito de 250 folios escrito a dos caras, contiene un sinfín de recetas e instrucciones para iluminar libros y pintura en general en los idiomas alemán y latín. La letra cambia con frecuencia, lo que hace pensar en un número elevado de autores.

Las dos únicas fechas que aparecen en su interior 1503 y 1508 , se encuentran casi a final del manuscrito; la primera de ellas, una indicación de Wolfgang (página 206), y la segunda, de Joh. Höflin de Augsburgo (página 228).

A partir de la página 100 del este manuscrito cambia no sólo la letra, sino también el concepto; en lugar de recetas, nos encontramos con un tratado que, pese a su volumen reducido, recuerda al tratado de C. Cennini, no sólo por el estilo, sino también por el contenido, ya que se describen técnicas de adorno (con estaño y matriz) que sólo se conocían por la obra de Cennini. 
Se han consultado tres fuentes donde aparece transcrito, traducido o citado aquellos apartados del El Liber Illuministarius, que describen la técnica del brocado aplicado [Von dem stanniol (Sobre la lámina de estaño) y Also nutz das stanniol (Usa la hoja de estaño como sigue)]; la primera de ellas una transcripción del texto original ${ }^{18}$, la segunda una traducción al inglés 19 y por último una traducción al alemán actual 20.

Estos documentos han servido de base para realizar la traducción al español de los dos apartados que describen la técnica del brocado aplicado y que presentamos a continuación por primera vez en este artículo ${ }^{21}$. Para ello se ha confrontado tanto el texto de origen ${ }^{22}$, como las traducciones comentadas, intentando reflejar de la forma más fiel posible, tanto su contenido como las diferencias terminológicas existentes entre las fuentes consultadas, sin pronunciarse en ningún momento sobre cuál es la versión más idónea, ya que se ha tenido en consideración las dificultades que entraña la traducción del texto original escrito en alemán antiguo y las posibles interpretaciones que se pueden realizar de algunos términos artísticos o acepciones.

\section{Acerca de la hoja de estaño}

Coge la hoja de estaño y antes de estamparla prepara el blanco como sigue: Raspa creta (carbonato de calcio) añade resina ${ }^{23}$ hasta que se note por el olor y muélela con agua y házla bastante espesa, pónla en un crisol y tápala con un paño húmedo para que no se endurezca; entonces coloca el bloque (matriz o molde ${ }^{24}$ ), cortado de antemano, y coge la hoja de estaño en la cantidad que quieras y deposítala sobre la matriz y frota bien en superficie con una esponja humedecida. Haz entonces una borla de estopa y humedécela bien, cógela por un extremo y colócala sobre la hoja de estaño y golpéalo sobre la estopa con un mazo hasta que la hoja de estaño penetre bien en la matriz; y cuando tu quites la estopa frota la hoja de estaño con el dedo hasta que notes el dibujo sobre el; y cuando tu hayas golpeado una parte o la totalidad del bloque, entonces coge un cuchillo cargado del mencionado blanco y extiéndelo con el cuchillo y ve suavemente con el cuchillo hasta que el blanco penetre solamente en los huecos. Después de esto, raspa con un cuchillo entre la hoja de estaño y el bloque soplando bastante bien sobre el y dejalo secar. Y si tu deseas tener más, entonces haz más hasta que tengas suficiente; pero si tu quiere cubrirlo con oro haz como sigue: coge un huevo y prepáralo como sigue: coge la clara y la yema del huevo y viértelas en un plato y bátelas con una pieza de madera hasta que se mezcle bien. Inmediatamente después deposítalo sobre la hoja de estaño, que debería estar situada frente a ti y extiéndela espesamente para que no pueda salirse y deposita el oro musivo 25 (Zwischt gold) donde ha sido aplicado; y si también tocas el relleno 26 no es dañino, y si se seca, quita el relleno.

Igualmente el relleno que depositas sobre la hoja de estaño lo puedes hacer con pintura a la cola o pintura al aceite.
Igualmente para obtener un relleno rojo coge bermellón y aceite, para uno azul coge un verde-azul o "lazur" 27 y témplalo con aceite; para un relleno marrón coge un rojo oscuro y agua de cola, para uno verde coge verdigris y aceite y muélelos muy bien y hazlo bastante fino, entonces se vuelven algo transparente.

\section{Usa la hoja de estaño como sigue}

Igualmente en tabla, o escultura o sobre tela hecha con pintura a la cola o las que todavía están sin preparar, remoja la tabla con cola tres veces y tu no necesitas mojar con cola la tabla o la escultura que ha sido cubierta con un fondo blanco ${ }^{28}$. Inmediatamente después coge cola

\section{Ficha 2. MANUSCRITO DE TEGERNEE}

\section{FICHA TÉCNICA:}

BROCADO APLICADO.

\section{FUENTE:}

EL LIBER ILLUMINISTARIUS O MANUSCRITO DE TEGERSNSEE.

TIPOLOGÍA:

Pintura tela, muro, escultura en madera

\section{MATERIALES:}

- Estaño batido en hojas.

- Dorado: ¿Oro musivo, u oro intermedio? y huevo (clara y yema)

- Pasta de relleno: Adhesivo: resina y Carga/pigmento: carbonato de calcio.

- Estopa.

- Color: bermellón, verdigris o azul ultramar aglutinado con aceite y rojo oscuro con agua de cola.

\section{MÉTODO DE EJECUCIÓN:}

\section{Proceso:}

- Preparación de la matriz (No se indica la naturaleza)

- Preparación de la masa de relleno: Moler creta raspada mezclada con resina y agua ponerla en un crisol y cubrir con paño húmedo para que no endurezca.

- Grabado de la lámina de estaño: Depositar sobre la matriz el estaño y frotar su superficie muy bien con una esponja humedecida. Colocar encima la estopa humedecida previamente y golpear con un mazo para obtener la impresión del dibujo de la matriz en la hoja de estaño.

- Eliminar la estopa y pasar el dedo sobre la superficie para comprobar si se ha impreso bien el dibujo.

- Aplicación de la masa de relleno: Extender la masa sólo sobre las incisiones del estaño. Cuando haya agarrado con un cuchillo se separa el estaño de la matriz y se deja secar.

Dorado de la hoja de estaño: El dorado se realiza con ¿oro musivo u oro intermedio? aplicado sobre la hoja de estaño preparada previamente con clara y yema de huevo bien batida y extendida en capa espesa.

Aplicación del color: Pintura a la cola 0 al aceite, los pigmentos que cita son el verdigris, azul ultramar, bermellón y rojo oscuro.

\section{APLICACIÓN DE LA HOJA DE ESTAÑO SOBRE LA OBRA:}

La hoja de estaño se pega en las obras con cola o con una pasta de harina y resina en polvo. Las obras que no han recibido un estrato de preparación se han de preparar previamente con tres capas de cola y las que si lo han recibido, se pegan directamente sobre ella con los materiales mencionados. 
$\longleftarrow$
$\square$ de madera y extiéndela en la hoja de estaño y empástala o haz una pasta de harina y polvo de resina en tanta cantidad como quieras y mézclalas juntas con un trozo de madera y extiéndela sobre la hoja de estaño y entonces empástala y pégalo donde quieras, o sobre una estatua cubierta de un fondo blanco, o sobre muros o telas, o pégalo también sobre aquello hecho con óleo. Coge el color dorado y úntalo / píntalo sobre la lamina de estaño y pégalo después y recuerda como regla general: donde vayas a aplicar o pegar pintura dorada $u$ otras pinturas ¿de barniz ${ }^{29}$ ? o pintura al óleo, embadurna de aceite el óleo, y embadurna de aceite ¿corrosivo ${ }^{30}$ ? el muro y el hierro; algunos preparan otra cosa para pegar la lamina de estaño en un muro: coge cal y mezcla con aceite y con barniz y unta /pinta con eso la lamina y pégala en el muro.

Para poder comparar las dos fuentes de esta técnica entre si, los datos de mayor interés de este manuscrito se encuentran resumidos en la ficha 2 .

\section{Estudio comparativo entre las fuentes escritas}

Tras el análisis de estas dos fuentes, el tratado de Cennini y el Manuscrito Tegernsee, es importante efectuar un estudio comparativo del proceso técnico y de los materiales citados. Los resultados de este estudio se exponen en la tabla $n^{\circ}$ I.

\section{El brocado aplicado: la literatura especializada}

La bibliografía consultada coincide en que el proceso de construcción del brocado aplicado es muy complejo, siendo básicamente el siguiente:

El motivo a reproducir se graba sobre una plancha de madera o metal de forma cuya morfología, según los casos, era cuadrangular o rectangular. Esta plancha denominada matriz servía para reproducir las veces deseadas el motivo a aplicar. Las dimensiones de la matriz es muy variada, por lo general oscila entre los $2 \mathrm{~cm}$ a los $20 \mathrm{~cm}$ de lado ${ }^{31}$. Hasta el momento no se conoce ninguna matriz por lo que las incógnitas en cuanto a su naturaleza material siguen hoy día sin despejarse.

Sobre esta plancha, se deposita una o varias lámina/s de estaño y se procede a realizar el estampado del dibujo de la matriz en la lámina de estaño. Este paso se realiza presionando o golpeando (con un mazo o similar) su superficie de forma homogénea, interponiendo para ello, un material de relleno (estopa) que sirve para amortiguar y transmitir de manera uniforme la presión ejercida, de tal forma que quedase impreso sobre ella, en negativo, el dibujo inciso en la matriz.

\section{Tabla I}

BROCADO APLICADO: ESTUDIO COMPARATIVO DE LAS FUENTES TRADICIONALES ESCRITAS (Tratados)

\section{TRATADO DE CENINNO CENINNI}

NATURALEZA DEL MOLDE:

Piedra

NATURALEZA MASA DE RELLENO:

Yeso grueso molido y cola

\section{PROCESO DE ESTAMPADO}

- Untar el molde con grasa o sebo

- Colocación del estaño

- Colocación de la estopa mojada

- Batir con maza de madera de sauce

- Eliminar la estopa

APLICACIÓN DE LA MASA DE RELLENO:
Extender solamente en los huecos dejados

DORADO DE LA HOJA DE ESTAÑO:

Aplicación de la mixtión sobre la hoja de estaño

Dorado con pan de oro fino

APLICACIÓN DEL COLOR:

No se indica

APLICACIÓN DE LA HOJA DE ESTAÑO SOBRE LA OBRA:

No se indica

\section{MANUSCRITO DE TEGERSNSEE}

NATURALEZA DEL MOLDE:

No se especifica ¿Piedra madera?

NATURALEZA MASA DE RELLENO:

Creta (Carbonato de $\mathrm{Ca}$ ), Resinas y agua molidas

PROCESO DE ESTAMPADO:

- Colocación del estaño

- Frotar la superficie con esponja humedecida

- Colocación de la estopa moojada

- Batir con maza de madera de sauce

- Eliminar la estopa y comprobar pasando el dedo que la impresión es correcta

APLICACIÓN DE LA MASA DE RELLENO:

Extender solamente en los huecos dejados, una vez seca,separar el estaño de la matriz y dejar secar.

DORADO DE LA HOJA DE ESTAÑO:

Aplicación de la clara y yema de huevo bien batida en capa espesa sobre el estaño.

¿Oro musivo u oro intermedio?

APLICACIÓN DEL COLOR:

TÉCNICA: Pintura a la cola 0 al aceite

PIGMENTOS: verdegris, azul ultramar, bermellón y rojo oscuro.

APLICACIÓN DE LA HOJA DE ESTAÑO SOBRE LA OBRA:

OBRAS SIN PREPARAR:

- Encolado de cola en 3 capas.

- Pegado del estaño con cola o pasta de harina y resina en polvo

OBRAS CON PREPARACIÓN:

Pegado del estaño con cola o pasta de harina y resina en polvo 
Sobre la parte exterior de la lámina de estaño, se vierte o extiende en estado semilíquido, la masa de relleno (también denominada masa de impresión) que llena únicamente los huecos dejados por el dibujo grabado en la matriz. Esta masa cumple varias funciones, en primer lugar al rellenar los huecos permite obtener el efecto de relieve de la decoración (hilos de oro y motivo reproducido), y en segundo lugar, una vez enfriada, facilita la separación de la lámina de estaño de la matriz sin que se rompa el estaño ni se deteriore el dibujo.

La composición de la masa varía en función de las escuelas. Los resultados analíticos de las obras examinadas o intervenidas con metodología cientííca, han dado hasta el momento dos tipos de masa diferentes, en función de la naturaleza de los componentes empleados 32:

Grasa: Compuesta principalmente de cera mezclada con miel, resina o aceite de linaza, algunas veces pigmentada.

Magra: A base de cola proteica (animal o huevo) y carbonato de calcio, algunas veces emulsionada con aceite y también a veces pigmentada.

Aunque también nos encontramos en la bibliografía consultada referencias a otros tipos de masas de impresión ${ }^{33}$

I Cera.

2 Cera-resina.

3 Cola y carbonato de calcio.

4 Cola, carbonato de calcio y fibras de papel.

5 Láminas de estaño-cola-carbonato de calcio.

Una vez concluido este proceso, se separa la lámina de estaño de la matriz y/o se corta el motivo, tras lo cual, se aplica sobre la obra empleando para ello un adhesivo, por lo general, compuesto por aceite, resina y cola proteica con o sin adición de pigmentos. La excepción la constituye los casos en los que los motivos eran aplicados directamente sobre una corladura en estado mordiente; en estos casos la propia naturaleza material de la corladura sirve de adhesivo.

En cuanto a la puesta en obra existen dos métodos diferentes, sobre la obra en blanco, es decir sobre la preparación, (brocados yuxtapuestos), o sobre la obra acabada (piezas de brocado).

El dorado se realiza siempre a mixtión con objeto de no alterar los relieves característicos de esta técnica durante su aplicación, por lo que su aspecto superficial es por tanto mate. El dorado de las piezas se podía realizar in situ sobre la obra, cuando los motivos eran aplicados sobre la obra sin acabar, es decir sobre la preparación o imprimación (brocados yuxtapuestos) o antes de ser aplicados sobre la obra acabada (piezas de brocado).

El último paso del proceso, de carácter optativo, consiste en rellenar del color deseado (generalmente rojo, azul o negro) las zonas de reserva existentes entre el motivo y/o el rayado del relieve que imitan los hilos de oro, contribuyendo de esta forma a aumentar el efecto de imitación y percepción táctil de los tejidos de brocados a los que intenta emular esta técnica.

\section{Tipologías}

Son dos las tipologías en las que podemos encontrarnos brocados aplicados de forma aislada o conjunta:

Foto $\mathrm{n}^{\circ}$ I. Brocado aplicado yuxtapuesto o continuo. Detalle del vestido del "Dios Padre". Ático del Retablo de los Santos Juanes. Capilla Real de Granada.

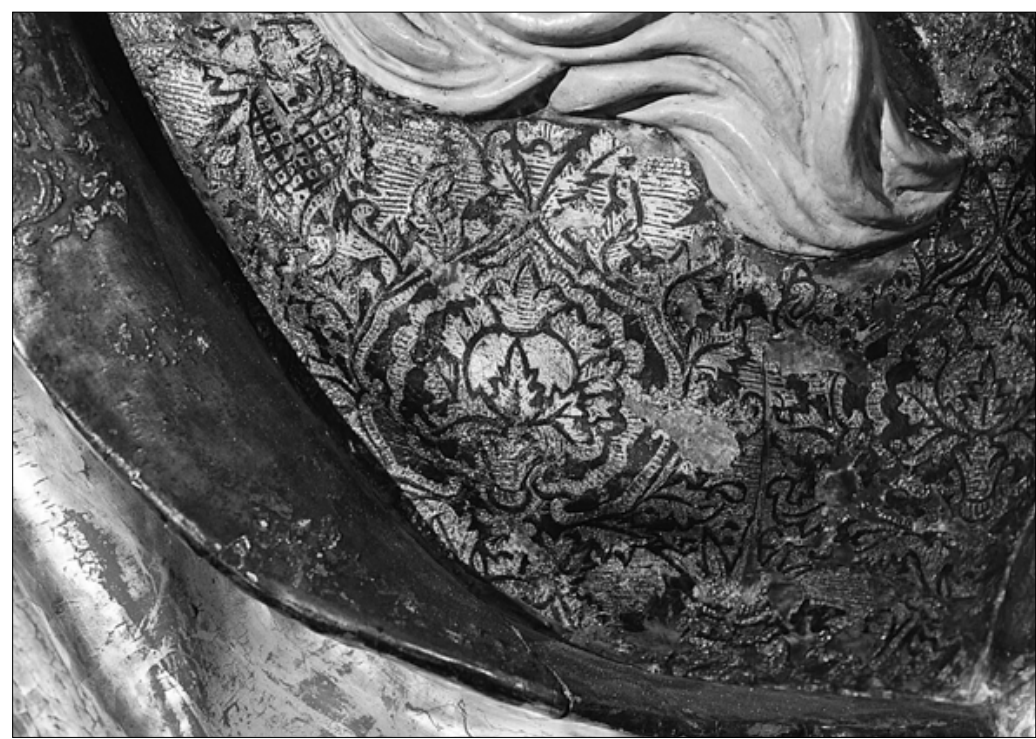

Dibujo N 1. Reconstrucción a partir de un módulo

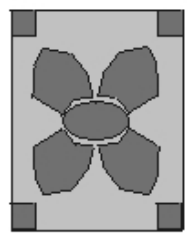

Módulo

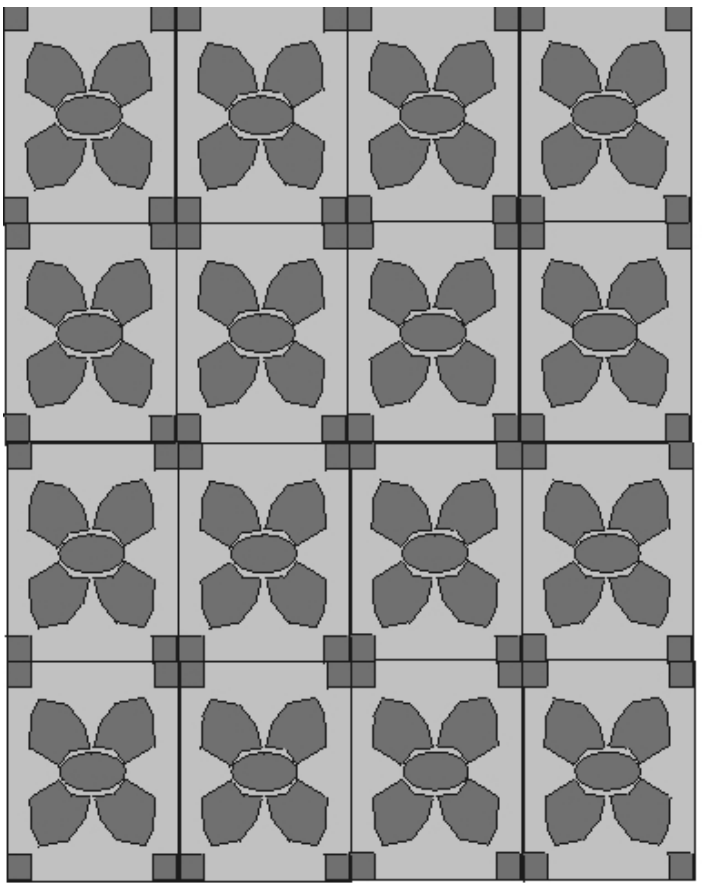


$\longleftarrow$
$\square$
$\square$

Foto $\mathrm{n}^{\circ}$ 2. Pieza de brocado o motivo aplicado. Detalle de la corona que decora la túnica del "Cristo con la cruz a cuestas". $2^{\circ}$ cuerpo del Retablo de los Santos Juanes.

Capilla Real de Granada

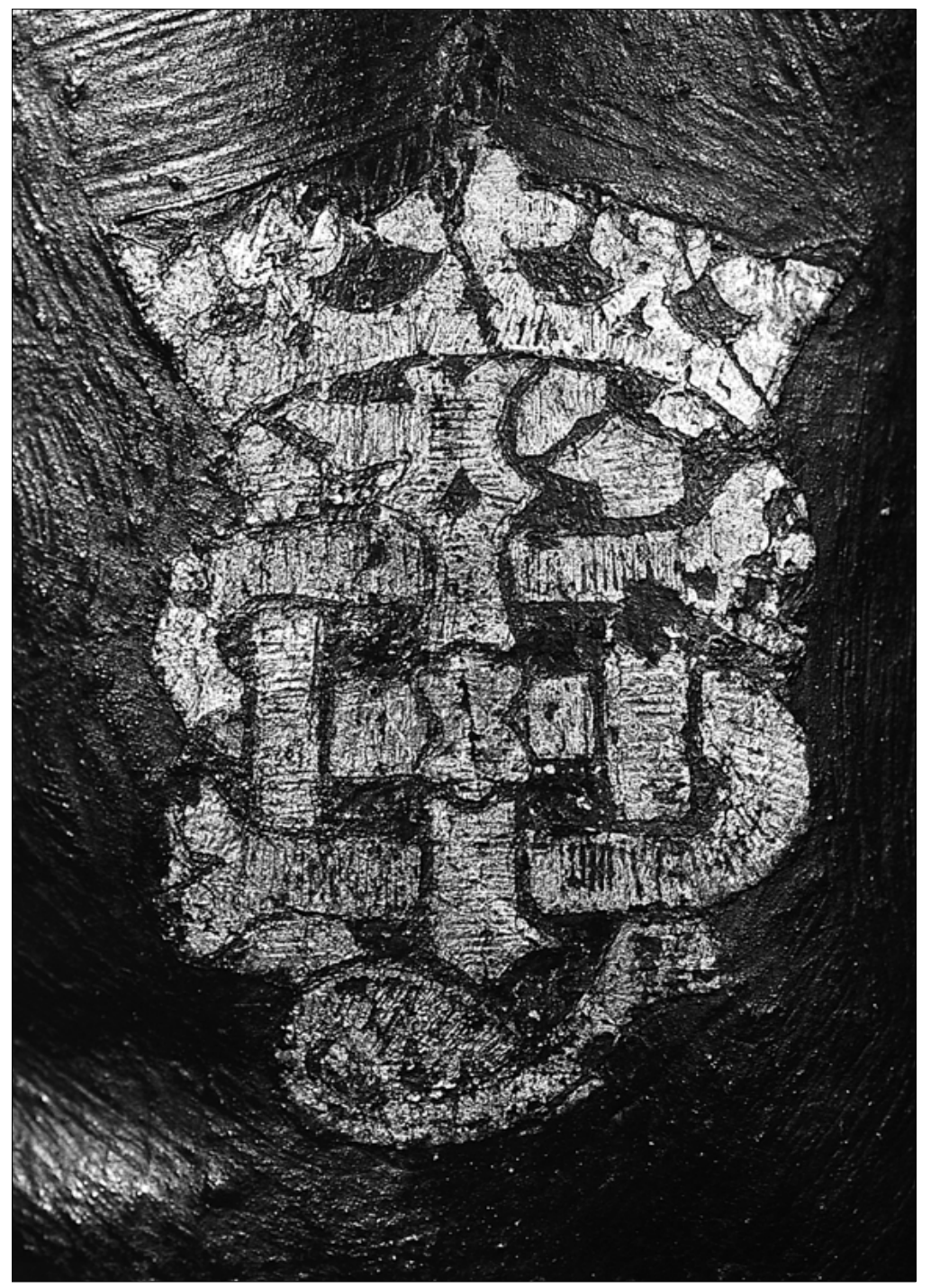

Brocados continuos: Motivos de forma rectangular o cuadrangular colocados de forma yuxtapuesta (uno al lado del otro). Se disponen sobre la preparación o imprimación de la obra a la que se adhieren con el adhesivo ya comentado. Una vez aplicado los motivos sobre toda la superficie se dora a la mixtión y/o se colorea. En general imitan tejidos con decoración uniforme (Véase dibujo $n^{\circ} \mid$ y foto $n^{\circ} \mid$ ).

Piezas de brocado/ motivos aplicados: Se refieren a los elementos sueltos que imitan bordados sobrepuestos a los tejidos. Técnicamente se resuelven aplicando los motivos seleccionados, por lo general motivos complejos con intricados contornos (águilas, granadas, follaje o bordes estrechos), sobre áreas específicas de la obra (por ejemplo, partes concretas de las vestiduras tales como las vueltas del manto). El dorado de estos motivos se realiza siempre antes de ser aplicados sobre ella (Véase foto $n^{\circ} 2$ ).

\section{Desconocimiento de la técnica: causas}

Son varias la causas que han contribuido al desconocimiento de esta técnica de decoración. En primer lu- gar, al mal estado de conservación, en el que por lo general han llegado hasta nuestros días los ejemplares conocidos que conservan este tipo de decoración, motivado por la propia fragilidad de estas técnica, por su naturaleza material y por la propia evolución de los numerosos materiales que intervienen o pueden participar en su composición.

En segundo lugar, debido a las modificaciones que han sufrido las policromías o las obras en su conjunto, fruto de cambios de gustos, modas o de estilos artísticos, que dan como consecuencia directa cambios sustanciales en su aspecto externo (repolicromados principalmente).

En tercer lugar, motivado por los tratamientos de conservación-restauración no adecuados que han sufrido algunas obras (eliminación de repolicromías, limpieza de la superficie pictórica, fijación de estratos con métodos no adecuados -calor y/o presión-, inmersiones en consolidantes, etc.), debido al desconocimiento de la técnica de ejecución y también de su naturaleza material, lo que ha implicado que se hayan intervenidos con tratamientos considerados "usuales" que, sobre otro tipo de técnicas polícromas han funcionado correctamente, pero que aplicados en esta técnica en concreto, tienen consecuencias nefastas para la conservación de sus características originales.

A estas causas conviene añadir, la dificultad de acceder a la bibliografía especializada y los pocos casos en los que se puede estudiar los brocados aplicados con una metodología técnica y científica específica que permita individuar casos concretos, intercambiar información entre especialistas, y promover su mejor conocimiento y divulgación.

\section{Conclusiones}

Como hemos visto el brocado aplicado es una técnica de decoración de gran calidad técnica, histórica y artística, cuyo conocimiento en los círculos especializados es relativamente reciente. Este artículo ha intentado abordar el estudio de esta técnica a partir del análisis de las fuentes escritas, con un doble objetivo: conocer la naturaleza material y al proceso constructivo y difundir en nuestro idioma tanto su contenido como las incógnitas que plantea su lectura.

Las conclusiones están por extraer y sólo se podrán obtener mediante las contribuciones que podamos aportar todos y cada unos de los especialistas que tenemos la oportunidad de enfrentarnos a obras con estas decoraciones. Sólo nos queda abordar el reto de afrontar su estudio empleando una metodología científica y rigurosa que permita mediante el intercambio de información, o la difusión de estudios o actuaciones, construir el camino que nos lleve a su conocimiento pleno. 


\section{Agradacimientos}

Desde aquí quiero hacer extensible mi más sincero agradecimiento a Agnes Le Gac y a Rosaura García por su desinteresada colaboración, a José Manuel Santos Madrid por su disponibilidad para documentar fotográficamente los brocados aplicados del retablo de la Capilla Real de Granada, a Rosario Villegas Sánchez, por su colaboración en la traducción del ingles del manuscrito de Tegernsee y muy especialmente a Barbara Stocker, sin cuyo trabajo no hubiese sido posible la traducción al español de este manuscrito.

\section{Documentación fotográfica}

Las fotografías que ilustran este artículo son propiedad de los fondos fotográficos del Instituto Andaluz del Patrimonio Histórico y han sido realizadas por el fotógrafo Sr. D. José Manuel Santos Madrid.

\section{Bibliografía}

\section{TRATADOS}

MANUSCRITO DE TEGERNSEE. "Liber Illuministarius" Munich Library, cod. Germ.82I

E. BERGER. Quellen und Technik der Frescko-, Oel-und TemperaMalerei des Mittelalters, 1912, nueva edición 1973, Dr. Martin Sändig oHg.

CENINNO CENNINN

- Tratado de la Pintura El Libro del Arte. Ed. E. Messeguer. $4^{a}$ ed. 1979, p: 76-79

- Il Libro del'Arte o Trattato della Pittura. A cura di Fernando Tempesti. Ed. Longanesi \& C, Milano. 1975

\section{Europea}

A.A.V.V. Gilded wood. Conservation and History. Sound View Press. Madison Conneticut. 1991.

A.A.V.V. Sculptures médiévales allemandes: conservation et restauration. Actes du colloque, Musée du Louvre, 6-7 décembre |99|. Ed Guillot de Suduiraut, Paris 1993

A.A.V.V. S.O.S. polychromies: dorures, brocarts et glacis. Namur. Mu sée des arts anciens du Namurois, 27 octobre-3I décembre. 1995. Foundation Roi Baudoin. Bruxelles, Belgium. IRPA. 1995.

BACHMANN. K.W., OLLERMANN. E. ET TAUBERT. J "The Conservation and Technique of the Herlin Altarpiece (1466)" en Studies in Conservation, 15, 1970, p: 329-369.

BALLESTREM. A. "Un témoin de la conception polychrime des retables bruxellois au début du XVle siècle" en Bulletin de Institute Royale du Patrimoine Artistique, Bruxelles X, 1967-68, p: 36-45.

BROECKMAN-BOKSTIJN. M., ASPEREN DE BOER J.R.J., VAN'THULEHRNREICH E.H. ET VERDUYN-GROEN. C.M. "The Scientific examination of the Polychromed Sculpture in the Herlin Altarpiece" en Studies in Conservation, I5, 1970, p: 370-399

COLINART, S. Y GILLOT DE SUDUIRAUT. S. "Un ejemplo de decoración en relieve: Los brocados aplicados. "La Natividad", bajorrelieve de madera de tilo polícromo (Colmar, 15 10, aprox.)" en revista KERMES, n³4, enero-abril 1999, p: 61-62.

COLINART, S. ÉVENO, M. "La polychromie: les etudes de laboratoire" en Sculptures médiévales allemandes: conservation et restauration. Actes du colloque, Musée du Louvre, 6-7 décembre |99|. Ed Guillot de Suduiraut, Paris 1993.

COREMANS y otros. La technique des primitifs Flamandes. Brussels. 1952-54.

DIDIER. R., KOCKAERT. L. , LOBELLE. H., SERK -DEWAIDE. M. "Le Saint Corneille sculpté de l'hôpital Saint-Jean à Bruges" en Bulletin de Institute Royale du Patrimoine Artistique, Bruxelles XX, 1984 85, p: || $7-|3|$

FRINTA. M. S. "The use of wax for Applique Relief Brocade on Wooden Statuary" en Studies in Conservation, Nº, 1963, p: 136-147

GETTENS R. J. AND. STOUT. G. L. Painting Materials. New York, |966, p: |49-|5|

HECHT, B. "Betrachtungen über Pressbrokate, Rekonstruktion versuche unter besondere Berúchsichtigung des sog Tegernseen Manuskript" en Maltechnik/Restauro 86 (I) (1980), P: 22-49
KOLLER. M. The Madonna of Friesach and her early dud late Gothic Polychromy. Reunión del Comité del ICOM para la Conservación. Madrid 1972

- "Leaf-Gilded Surfaces: Burnishing and Varnishing in Central Europe" en A.A.V.V. Gilded wood. Conservation and History. Sound View Press. Madison Conneticut. 1991, p: 291-300.

MASSCHELEIN-KLEINER. L. y ... ... "Contribution á I'analyse des liants, adhesives et vernis anciens". Studies in Conservation, 13. 1968

MILLS. J. AND PLESTERS. J. "Analysis of Wax Appliqué" en Studies in Conservation, 8, 1963, p: I47-148

NIEUWDORP, H.M.J. AND ANNAERT, M. "Het laat-1 4de eeuwsMariabeeld uit Brugge in het Museum Mayer van den Bergh en haar polychromie" en Bulletin de Institute Royale du Patrimoine Artistique, Bruxelles XVI, 1976-77, p: 26-36

OELLERMANN, E. "Zur Imitation textiler Strukturen in der spátgotischen Fass- und Flachmalerei," [The imitation of textile structures in late Gothic painting], Berichte des bayerisches Landesant dür Denkmalpflege 25. 1966, p: 159-174.

PERUSINI. T Y PERUSINI. E. "La tecnica del Pressbrokat nell'altare de Giovanni Martini a Remanzacco" en Restauro nel Friuli Venezia Giulia. Memorie della Scuola Regionale di Restauro, nº 9. I. 1983.

PERUSINI. T, PERUSINI. E. Y BERGAMINI G. L'altare ligneo di Giovanni Martini a Remanzacco. Remanzacco. 1986

PERUSINI, G. "Il Flügelaltar di Santa Fosca a selva di Cadore: storia, tecniche e restauro" en Zeitschrift für Kunsttechnologie und Konservierung. 1996.

PORTELL. J. D. "Altered silver Gilgding" en A.A.V.V. Gilded wood. Conservation and History. Sound View Press. Madison Conneticut. |99|, p: 205-216.

SCHLOSSER. J. La literatura artística. Ed Cátedra.3ª ed. 1976.

SERK-DEWAIDE. M

- "La datation de la polychromie d'una Vierge à I Enfant du Bohême révélée par l'examen" en Bulletin de Institute Royale du Patrimoine Artistique, Bruxelles XIV, 1973-74, p: I21- 127

- "Retable domestique à volets peints" en Bulletin de Institute Royale du Patrimoine Artistique, Bruxelles XVIII, 1980-8I, p: 23031

- "Decors en reliefs approche technologique et historique" en El retablo d'Issenheim et la scuplture au Nord des Alpes a la fin du Moyen Age. Actes du Colloque de Colmar (2-3 november 1987). Boletin de la Societé Schongauer, 1989. P: 91-97

- "The History and Conservation of the Surface Coating on European Gilded-Wood Objects" en A.A.V.V. Gilded Wood. Conservation and History. Sound View Press. Madison Conneticut. 1991, p: 65-78

- "Relief Decoration on sculptures and paintings from the thirteenth to the sixteenth century: technology and treatment". Dossier IRPA. Congress 1991. 
SERK-DEWAIDE M. AND VERFAILLE. S. "De "Virga Jessé" van Hasselt, Voobeeld van een I 4de stoffering" en Bulletin de Institute Royale du Patrimoine Artistique, Bruxelles XVII, 1978-79, p: I28137

Española

A.A.V.V. Mirari. Un pueblo al encuentro del arte. Catálogo de la Exposición en la Sala Amárica. Diputación Foral de Álava, Vitoria. 1989, p: 449-453

A.A.V.V. "Restauración del Retablo renacentista de Bidaurreta". ARTELEKU. Diputación Foral de Gipuzkoa. Departamento de Cultura, Educación, Deporte y Turismo. San Sebastián 1991, p: 64-7|

ANTÓN, LUIS CRISTOBAL "Restauración del Retablo Mayor de la Iglesia de Santiago del Arrabal de Toledo". Pátina nº 7, Madrid, 1995, P: 18-36

AZCÁRATE, J. M. Ars Hispaniae XIII. Madrid 1958

CORESAL Restauración del retablo de San Miguel, Ezcaray. Folleto editado por el Grupo Coresal.
ESTRADE, Ma DE LOS MILAGROS Brocado aplicado una técnica insólita empleada en el retablo de Santa María de Galdakao (Bizkaia). VII Congreso de Conservación de Bienes Culturales, 23, 24 y 25 de septiembre. Universidad de Deusto. Bilbao. Ed. Gobierno Vasco. Departamento de Cultura y Turismo. Vitoria, 1991, p: I26136.

GÓMEZ MORENO. Ma. E. La policromía en la escultura española. Madrid 1943

GÓMEZ, C., SALAZAR J. A. Y DE MIGUEL J. Brocado aplicado: Nuevas aportaciones. $X$ Congreso de Conservación de Bienes Culturales, 29 de septiembre a 2 de octubre de 1994, Cuenca, p 28I291.

GÓMEZ, M ${ }^{\mathrm{a}}$ L. Examen científico del Santo Entierro de Granada atribuido a Jacopo Torni. X Congreso de Conservación de Bienes Culturales, 29 de septiembre a 2 de octubre de 1994, Cuenca, p: 263-272

\section{GARCÍA RAMOS, R. Y RUÍZ DE ARCAUTE, E.}

- Aproximación al brocado aplicado en España. Desarrollo y extensión. XI Congreso de Conservación de Bienes Culturales, del 3 al 6 de octubre de 1996, Castellón, p: 747-756.

- "El brocado aplicado una técnica de policromía centroeuropea en Álava" en Eusko Ikaskunza n 17. Donostia 1988, p: 409-421

Notas

I. FRINTA. M. S. "The use of wax for Applique Relief Brocade on Wooden Statuary" en Studies in Conservation, 8, 1963, p: I36-147

2. Véase página $n^{\circ} 68$ de Myriam Serk-Dewaide "The History and Conservation of the Surface Coating on European Gilded-Wood Objects" en A.A.V.V. Gilded Wood Conservation and History. Soud View Press. 1991, página: 65-78

3. Véase páginas 88-92 de Franceschi F. "Poder, lujo y terciopelo" en Revista La aventura de la Historia. Año 2, n 16, 2000.

Véase página 136 de Mojmír. S. Frinta "The use of Wax for Appliqué Relief Brocade on Wooden Statuary" en Studies in Conservation, $n^{\circ}$ 8, 1963, p: |36-147.

4. Véase página $n^{\circ}$ I de HECHT, B. "Betrachtungen über Pressbrokate, Rekonstruktion versuche unter besondere Berúchsichtigung des sog Tegernseen Manuskript" en Maltechnik/Restauro 86 (I) (1980), P: 22-49

5. Véase op. Cit. $N^{\circ} 4$.

6. Myriam Serk-Dewaide menciona el uso de esta técnica por pintores como el Maestro de Flémalle (La Virgen con Niño, Santa Verónica y El Mal Ladrón, Fráncfort/M. Städelsches Kunstinstitut, inv n 939, 939 A y 886), Jean Van Eyck (El Cordero Místico, Virgen, Dios Padre y Juan Bautista, catedral de Saint-Bavon, Gante) y Roger Van der Weyden, Políptico del Juicio Final, Beaune, Hôtel-Dieu, al igual que su empleo en la policromía de retablos de los Países-Bajos Meridionales. Véase página 91 de "Decors en reliefs approche technologique et historique" en El retablo d'Issenheim et la scuplture au Nord des Alpes a la fin du Moyen Age. Actes du Colloque de Colmar (2-3 november 1987). Boletin de la Societé Schongauer, 1989. P: $91-97$

7. Véase página $n^{\circ} 29$ de op. Cit, $n^{\circ} 4$

8. Cap: 24 de Theophilus On Divers Arts. The Foremost Medieval Treatise on Painting, Glassmaking and Metal work. Ed. Dover. $2^{\mathrm{a}}$ ed. 1979

9. Cap: XIII (276). "De coloribus et artibus romanorum" en M. Ph. Merrfield. Dover Inc, I a ed. 1967. Vol. I, p: 221 .

10. Realización de una serie de tratamientos superficiales de la lámina de estaño que permiten obtener el efecto especular y brillante característico, generalmente se consigue mediante su pulido y posterior bruñido.

I I. Cap: 205-209 de "De coloribus facendi" (Ms. De Jean le Begue) en M. Ph. Merrfield. Dover Inc, I ${ }^{a}$ ed. 1967. Vol. I, p: 205-209.

12. Véase $X C V, X C V I I, X C I C, C$, y Cl., de Tratado de la Pintura El Libro del Arte. Ed. E. Messeguer. $4^{\mathrm{a}}$ ed. 1979

Il Libro del'Arte o Trattato della Pittura. A cura di Fernando Tempesti. Ed. Longanesi \& C, Milano. 1975.
13. Véase cap. CXXVIII "De cómo se hacen algunos relieves y cómo sirven para el muro y la tabla" de op. Cit,, $n^{\circ} 12$

14. Véase páginas $n^{\circ} 100$ a 108 de MANUSCRITO DE TEGERNSEE. "Liber Illuministarius" Munich Library, cod. Germ.82I y páginas 194-195 de E. BERGER. "Quellen und Technik der Frescko-, Oel-und Tempera-Malerei des Mittelalters, 1912, nueva edición 1973, Dr. Martin Sändig oHg.

15. Véase página $n^{\circ} 97$ de Julius Schlosser La Literatura Artística. $3^{\text {a }}$ ed. Cátedra. Colección Arte Grandes Temas. 1976.

16. Véase op. Cit, $n^{\circ} 13$

17. De cómo se hacen algunos relieves y cómo sirven para el muro y la tabla

Aun te podrás servir de una piedra tallada por molde, de la forma que quieras, y untarla de lardo o saín. Después toma una hoja o pan de estaño batido y con ayuda de una muñeca de estopa mojada, poniéndola encima del estaño y éste encima de la impronta o molde, golpea con una maza de sauce cuanto puedas. Toma luego yeso molido con cola y con la espátula rellena (el hueco de) la impronta. Puedes con ello adornar el muro, los cofres, las piedras, lo que quieras, poniendo mordiente encima del estaño, y ciando esté a punto dorarlo con oro fino. Cuando esté seco lo pegas al muro con pez de calafate.

Come si fa alcuno rilievo tratto d'impronta di prieta, e come son buoni in muro e in tavola.

Ancora puoi avere una pietra, distagliata di divise di qual ragione che vuoi, e ungere la detta pietra con lardo o con sugna. Poi avere dello stagno battuto; e con stoppa alcuna cosa bagnata sopra lo stagno ch'è sopra la impronta, battendolo forte con uno magliuolo di salico, quanto puoi. Abbi poi gesso grosso macinato con colla, e con la isteca riempi questa cotale stampa. Ne puoi adornare in muro, in coffani, in prieta, e in ciò che vuoi; mettendo poi di mordente di sopra lo stagno; e quando morde un poco, metterlo d'oro fine. Attaccala poi al muro quando è secco, con pece di nave.

18. Véase, op. Cit. No 14

19. Véase página 388-89 de BROECKMAN-BOKSTIJN. M., ASPEREN DE BOER J.R.J., VAN'THULEHRNREICH E.H. ET VERDUYN-GROEN. C.M. "The Scientific examination of the Polychromed Sculpture in the Herlin Altarpiece" en Studies in Conservation, 15, 1970, p: 370-399

20. Véase op. Cit $n^{\circ} 4$

21. La traducción se ha realizado por Barbara Stocker, a la cual le agradezco su disponibilidad y amabilidad para colaborar conmigo en esta empresa.

22. VON DEM STANNIOL.

Nym das stanniol vnd e du das stempfs (starnpfest) so berait aín weyss also: reib kreyden, vnd pech dadrein als vil das man das 
wol darauss smek vnd reib das in leimwasser vnd mach das gar dick vnd tu das in ein tegl vnd leg ein nass tüchlin dadrüber das es nit hart wird, und nim dann den model der auss geraist sey, vnd nim das stanniol als vil du wilt vnd legs auf den model vnd schmer das gulden ein (sckmier es gut ein) vnd über fars mit einem nassen padswam. darnach rnach ein puschel aus werck (Werg) vnd netz das gar wol vnd nyms bei ainem zipfel vnd heb es auf das stanniol vnd schlag auf das werck mit ein klein schlege das das stanniol wol in den model kom vnd wenn du das werck auf hebst, so greiff mit ainen vinger auf das stanniol, das du das mit aufzüchst vnd wenn du ain tail geschlagn hast oder gar was auf dem model gewesen ist, so nin denn ein messer und das obgenant weyss vnd trags mit dem messer auf vnd far mit dem messer schön darüber heus, dass das weyss nur in die raisel (Vertiefungen). Darnach greiff mit einem messer zwischen das model und das stanniol und heus (blase) gar schön dadrauf vnd las es drucknen. Und wildu mer haben so mach sein mer pis tu sein genug hast und wiltu aber das überziehen mit golt (Gold), so tu das also: mach ein air klar also, nim das weis vnd den totter vnd schütt es in ein schüssel und tper. es mit einem holtz gar wol, dass es sich wol vermisch. Darnach streich es auf das stanniol das sol vor dir ligen vnd streich es als dick auf, das es nit herab mag gerínnen vnd scheuss denn zwischt golt (Rauschgold, unechtes Blattmetall) darein wo es geschlagen sei vnd ob es die feldung (Füllung) auch trifft das schad nit vnd wen dasselbig drucken wird, so trag die feldung auf.

Item die feldung auf das stanniol magstu machen ven leim varb oder von öl varb. Item zu roter veldung nim zinober vnd öl, zu plab (blau) nim ein grün plab oder lasur vnd tempirs mit öl, zu praun feldung nim ein tunkels rössl vnd leim wasser, zu grün nim spangrün vnd öl vnd reibs den gar wol vnd mach in gut dünn, so wird es etwas durchsichtig

\section{Also nutz das stanniol.}

Item auf tafel oder pild oder auf tuch dy mit leim varb gemach sey oder noch blass (blass) sein, so leimtrenks oder die tafel dreystund (dreimal) vnd die tafel oder pild, die geweist sey, die bedarfst du nit leimtrenken. Darnach nim holtz leiin und streich in an das stanniol vnd kleib es dann an oder mach ein kleystern von mel vnd darein ein pulver von pech als vil, dass man das wo smek vnd misch das untereinander mit einem holzlin vnd streich dann an das stanniol vnd kleib es dann an, wo du wild oder auf pild die geweist sind oder an meir (mäuer) oder an tüchern oder was mit öl gemacht ist, kleib es also an. Nim die golt varb vnd streichs an das stanniol und kleibs darnach an vnd merk für ain gemeinsame regl: wo du die golt varb oder ander fuerniss varb oder öl varb auf tregst oder ankleibst, so öl trencks vorhin mit öl vnd die meier vnd eysen trencks mit Baissen öl (Beitzenöl), etlich machent zu dem stanniol ein ander zeug auf die meir (Mauer) vnd also: nim kalisch (Kalk) vnd reib den mit öl vnd mit firniss vnd streychs (streich es) an das stanniol vnd kleib es dann an die maur.

23. En la traducción al inglés, se puede traducir como "... en la cantidad que quieras ..."

24. El término block (bloque) puede referirse a mármol o a madera.
25. Es en la traducción del término Zwischt gold donde existe mayor contradicción a la hora de traducir este tratado.

En la traducción al inglés (Véase página 389-390 de op. Cit. $N^{\circ}$ 19) se traduce por Mosaic gold, u oro musivo, también denominado con fines artísticos purpurina o mosaico dorado. Este material compuesto por estaño, plata, azufre y sal de amonio, era conocido en Europa a principios del s. XII como sustituto del oro. Aparece citado en textos clásicos del S. XIV y XV como el Manuscrito de Bolonia (Merrifield vol II, p.458), De arte lluminandi (F. Brunello, p 55), y en la literatura moderna, en el Thompson (The materials of Medieval Painting. Ed. Dover, p. 182-84), en La Fabbrica dei colori. Ed. II Bagattto. 1986, p:227-229, y en el Gettens \& Stout Painting Materials a short encyclopaedia. Ed. Dover .p. 132.

Por el contrario Brigitte Hecht lo traduce como oro intermedio (véase página 32 y notas nº 9 y 20 de op. Cit. N4). Refiriendose al material compuesto por oro por un lado y plata por el otro utilizado por los orfebres del oro (Archivo de Nürenberg, MS I I 8 disposición sobre los orfebres del oro). El oro intermedio se ha encontrado en la composición de brocados aplicados que, como en los casos del altar de Tiefenbronn ( 143 |/32 y en una parte del altar de Blauderen, se han realizados estudios analíticos sobre su composición.

26. La traducción al inglés (Véase página 389 de op. Cit. № 19) indica que se refiere "...probablemente el área del diseño del brocado destinada a ser pintada más tarde; la palabra "feldung" se utiliza otras veces como la pintura empleada para las áreas a rellenar"

27. Lazur: en el siglo XI Teófilo en la Schedula Diversarum Artium nombra un color, el lazur, que, en recientes estudios como el de A. Raft ("About Theophilus blue colour lazur", en Studies in Conservation, 1968, vol. XIII, p: I-6), se ha concluido que se refiere al azul ultramar. También aparece citado en La Fabbrica dei colori. Ed. II Bagattto. 1986, p: 308.

28. Se refiere a la capa de preparación.

29. Véase op. Cit. $N^{\circ} 21$.

30. Véase op. Cit. $n^{\circ} 21$

31. Tamaño de los casos conocidos

32. Véase:

- página 37 de Myriam Serk-Dewaide "Relief decoration on sculptures and paintings from the thirteenth to the sixteenth century. Tecnology and treatment". Dossier IRPA. Congress 1991,

- página 91 de "Decors en reliefs approche technologique et historique" en El retablo d'Issenheim et la scuplture au Nord des Alpes a la fin du Moyen Age. Actes du Colloque de Colmar (2-3 november 1987). Boletin de la Societé Schongauer, 1989. P: $91-97 y$

- página $n^{\circ} 68$ de "The History and Conservation of the Surface Coating on European Gilded-Wood Objects" en A.A.V.V. Gilded wood. Conservation and History. Sound View Press. Madison Conneticut. 1991, p: 65-78

33. Véase página $n^{\circ} 24$ op. Cit. $N^{\circ} 4$. 